\title{
Los procesos de lectoescritura de los mensajes de texto en redes sociales para la enseñanza de
}

\section{ELE}

\author{
Myriam Waked-Hernández ${ }^{*}$
}

Licenciada en Idiomas, Especialista en Archivística con énfasis en Administración, Docencia e Investigación. Docente, Facultad de Educación, Universidad Pedagógica y Tecnológica de Colombia, Tunja, Colombia.

Correo electrónico:

myriam.waked@uptc.edu.co.

Recibido: 3 de diciembre del 2015

Aprobado: 5 de febrero del 2016

Cómo citar este artículo: Waked-Hernández Myriam. "Los procesos de lectoescritura de los mensajes de texto en redes sociales para la enseñanza de ELE". Rastros Rostros 18.32 (2016): 67-76. Impreso. doi: http://dx.doi. org/10.16925/ra.v18i32.1319.

\section{Resumen}

Introducción: este artículo presenta los resultados de la investigación "Implementación del Nivel c1 del Marco Común de Referencia Europea para las Lenguas (2012)", la cual originó una de las secciones de Comunicarse en español: la mejor opción. Este libro se estructuró en varias secciones; la sección mencionada es “Comparta sus ideas con la red social". Metodología: para lograr el objetivo, se llevó a cabo una investigación-acción. El método fue inductivo, pues el uso de diversas lecturas y la escritura de los mensajes de texto permitieron que un extranjero usara las redes sociales con fines académicos. Los ejercicios se aplicaron a un grupo de cuatro estudiantes extranjeros: tres alemanes y una de la China, entre las edades de 18 y 23 años, interesados en aprender, mejorar y hablar el español como lengua extranjera (ELE). Resultados: se vio que los estudiantes extranjeros mejoraron los procesos de lectoescritura; esto se reflejó en el reconocimiento y la contextualización que los aprendientes de ELE demostraron en la realización de cada ejercicio de lectura y escritura a través de los mensajes de texto y las intenciones comunicativas que rodean su uso en contextos determinados. Conclusiones: de acuerdo con las indicaciones de los ejercicios de Comunicarse en español: la mejor opción en la etapa de "Comparta sus ideas en la red social", los estudiantes extranjeros, inicialmente, desarrollaron los ejercicios utilizando las redes sociales mediante la lectura y escritura. Esta investigación fue de gran aporte para los aprendientes en cuanto al manejo de la lengua española.

Palabras clave: competencia comunicativa, ELE, escritura, lectura, redes sociales, TIC. 


\title{
The Literacy Processes of Text Messages on Social Networks for the Teaching of ELE
}

\begin{abstract}
Introduction: This article presents the results of the research study titled "Implementation of the c1 level of the Common European Framework of Reference for Languages (2012)," which led to one of the sections of Communicating in Spanish: The Best Option. This book is divided into several sections, and the section mentioned is titled "Share your ideas with your social network." Methodology: An action research study was developed to achieve the aim. The method was inductive, as the use of a variety of readings and the writing of text messages allowed foreigners to use social networks for academic purposes. The exercises were applied to a group of four international students: three German students and one Chinese student between the ages of 18 and 23 interested in learning, improving, and speaking Spanish as a foreign language (ELE). Results: It was found that the international students improved their literacy processes: this was reflected in the recognition and contextualization that the ELE learners demonstrated when completing each reading and writing exercise through text messages and the communicative intentions surrounding their use in certain contexts. Conclusions: In adherence to the instructions of the exercises in the section titled "Share your ideas with your social network" in Communicating in Spanish: The Best Option, international students initially completed the exercises by using reading and writing on social networks. This research greatly contributed to the learners' command of the Spanish language.
\end{abstract}

Keywords: communicative competence, ELE, writing, reading, social networks, ICT.

\section{Os processos de lectoescrita das mensagens de texto em redes sociais para o ensino de ELE}

\section{Resumo}

Introdução: este artigo apresenta os resultados da pesquisa "Implementação do Nível C1 do Quadro Europeu Comum de Referência para as Línguas (2012)", a qual originou uma das seções de Comunicar-se em espanhol: a melhor opção. Esse livro estruturou-se em várias seções; a seção mencionada é "Compartilhe suas ideias com a rede social". Metodologia: para atingir o objetivo, realizou-se uma pesquisa-ação. $\mathrm{O}$ método foi indutivo, pois o uso de diversas leituras e a escrita das mensagens de texto permitiram que um estrangeiro usasse as redes sociais com fins acadêmicos. Os exercícios foram aplicados a um grupo de quatro estudantes estrangeiros: três alemães e uma da China, entre 18 e 23 anos, interessados em aprender, melhorar e falar espanhol como língua estrangeira (ELE). Resultados: percebeu-se que os estudantes estrangeiros melhoraram os processos de lectoescrita; isso se refletiu no reconhecimento e na contextualização que os aprendizes de ELE demonstraram na realização de cada exercício de leitura e escrita por meio das mensagens de texto e as intenções comunicativas que rodeiam seu uso em contextos determinados. Conclusões: de acordo com as indicações dos exercícios de Comunicar-se em espanhol: a melhor opção, na etapa "Compartilhe suas ideias na rede social", os estudantes estrangeiros, inicialmente, desenvolveram os exercícios utilizando as redes sociais mediante a leitura e a escrita. Esta pesquisa foi uma grande contribuição para os estudantes no que se refere ao uso da língua espanhola.

Palavras-chave: competência comunicativa, ELE, escrita, leitura, redes sociais, TIC. 


\section{Introducción}

En el presente artículo se entienden la lectura y la escritura como procesos metodológicos, ya que algunos ejercicios y actividades desarrolladas por un grupo extranjeros aprendientes del español incluyeron mensajes de texto con el fin de mejorar su proceso de aprendizaje del español como lengua extranjera. De Comunicarse en español: la mejor opción (Waked, Rodríguez, Aponte y Cardozo 18), se diseñó una de las secciones, "comparta sus ideas en la red social", en la cual se incluyeron ejercicios de lectura y escritura que facilitaron a los estudiantes extranjeros utilizar de forma adecuada el uso del lenguaje al momento de expresar sus ideas, opiniones, comentarios a través de los mensajes de texto.

En el nivel $\mathrm{C} 1 \mathrm{o}$ avanzado de Comunicarse en Español: la mejor opción (Waked et al., 2015), los aprendientes adquirieron una amplia fundamentación en lo relativo a la gramática, al léxico, al uso de las tecnologías de la información y comunicación, y en todo lo que se requiere para el desarrollo de las cuatro habilidades: hablar, leer, escribir y escuchar. De esta forma, las necesidades del aprendiente en nivel $\mathrm{C} 1$ tienen que ver con el mejoramiento de la comprensión, la interpretación y la producción de todo tipo de discursos orales y escritos. Con base en esto, se incluyeron actividades y ejercicios que permitieron el mejoramiento de su competencia comunicativa en ELE.

Se optó por un enfoque pragmático de comunicación que destaca, ante todo, la relación entre el texto, el hablante y la situación comunicativa. Teniendo en cuenta los fundamentos del enfoque pragmático, en este libro el mejoramiento en el nivel de la lengua se hace con base en actividades en las que el uso es protagónico tanto en interacciones directas como virtuales. Esto facilitó el diseño de ejercicios que involucraron los espacios cibernéticos, como los mensajes de texto en las redes sociales, los blogs, los chats, a través de la lectoescritura.

El propósito de esta investigación es aplicar actividades de lectura y escritura en los mensajes de texto por medio de las redes sociales que utilizaron algunos estudiantes extranjeros en diversas actividades. Para cumplir con este objetivo general, se diseñaron los siguientes objetivos específicos. Se profundizó en el uso de la lectoescritura en los mensajes de texto por las redes sociales que utilizaron los extranjeros para comunicarse eficazmente. Se buscó mejorar la competencia comunicativa de los aprendientes de ELE mediante el uso de la Web, la telefonía móvil, Internet y las tabletas por medio de diversas actividades de lectura y escritura. Se intentó resaltar el cambio de la forma lingüística por el soporte informático basado en el tipo de escritura que coloquialmente se usa en las redes sociales.

Para cumplir con los objetivos de la investigación, se tuvieron en cuenta temas como la pragmática y las redes sociales en la Web 2.0. De esta manera, las definiciones y comentarios de diversos autores fueron de gran ayuda para el desarrollo de esta investigación. A continuación se nombran los referentes teóricos y las definiciones de algunos autores.

\section{Referentes teóricos}

\section{Punto de vista pragmático}

La lingüística es la ciencia que se encarga de estudiar el lenguaje. Dentro de la lingüística surge la pragmática como una disciplina que se encarga de analizar la lengua en el uso. De esta manera, la pragmática tiene sus inicios en la filosofía del lenguaje. Escandell asegura que la pragmática es:

[...] el estudio de los principios que regulan el uso del lenguaje en la comunicación, es decir, las condiciones que determinan tanto el empleo de un enunciado concreto por parte de un hablante concreto en una situación comunicativa concreta, como su interpretación por parte del destinatario. (13-14)

Así mismo, Gutiérrez afirma que "[l]a [p]ragmática se presenta como la disciplina que tiene por objeto de estudio la comunicación lingüística en toda su extensión y profundidad" (95).

Por lo tanto, en esta investigación se tuvo en cuenta la disciplina pragmática con el fin de mejorar la lectura y la escritura de los aprendientes de ELE. Es así como la pragmática analiza la relación entre el hablante, el texto y la situación comunicativa. Además, dentro de la pragmática, se deben tener en cuenta los actos de habla y su relación con el contexto sociocultural colombiano. De esta forma, un acto de habla tiene que ver con las cosas que hacemos con el lenguaje, por ejemplo, preguntar, informar, ordenar, pedir, prometer, etc.

Las competencias pragmáticas, según el Marco Común de Referencia Europea para las Lenguas: 
[...] se refieren al conocimiento que posee el estudiante de los principios según los mensajes:

1. Competencia argumentativa (se organizan, se estructuran y se ordenan)

2. Competencia funcional (competencias comunicativas)

3. Competencia organizativa (se secuencian según esquemas de interacción y de transacción). (121)

Al aprender español, el estudiante extranjero le da mayor importancia a la coherencia y a la cohesión en el desarrollo de la competencia del discurso.

En conclusión, "[1]a pragmática se encarga de estudiar el uso que hacemos del lenguaje y el efecto que produce este en el oyente" (Lomas et al. 32). Este aspecto es de vital importancia para la investigación desarrollada, ya que orientó la construcción de un texto y su eficacia en el cumplimiento del propósito por el cual fue utilizado.

\section{Las redes sociales en la Web 2.0}

La Web 2.0, de acuerdo con Rosario Peña et al.:

[...] nos presenta una visión de Internet como plataforma de trabajo horizontal en el que el usuario puede adoptar un papel más activo para expresarse, opinar, recopilar, compartir información, colaborar y crear conocimiento utilizando páginas dinámicas con contenidos abiertos que nos suministran herramientas online y nos proporcionan espacios de trabajo cooperativo. (131)

En los últimos tiempos, el mundo de las comunicaciones experimenta una enorme transformación que afecta a los hábitos sociales y, entre ellos, a la manera de hablar, escribir, leer y comunicarse. Hoy en día que la población mundial puede acceder a la información, a la comunicación y a las redes sociales, las posibilidades para acceder a otras realidades culturales diferentes a la propia se multiplican, con lo que una parte de la población mundial puede acceder con facilidad a otras culturas y a otros idiomas.

Las novedades en el campo de la información y la comunicación permiten ponerse en contacto con el otro lado del planeta en décimas de segundo.

Las redes sociales, social networking sites en inglés, son básicamente:
[...] herramientas telemáticas de comunicación que tienen como base la web, que permiten a un usuario crear un perfil de datos sobre sí mismo en la red y compartirlo con otros usuarios. Conectan sucesivamente a los propietarios de dichos perfiles a través de categorías, grupos, etiquetados personales, ligados a su propia persona o perfil profesional. (Castañeda 25)

Tener una red de conocidos, lo que ya llamamos networking, permite acceder a otros puntos de vista o a diferentes posiciones de influencia.

Para Calvo y Rojas, las redes sociales virtuales constituyen:

[...] una herramienta fundamental de networking, lo cual significa trabajar con redes, estas redes pueden ser desde redes informáticas hasta lo que hoy se conoce como redes sociales, implica la habilidad de conectar con varios tipos de recursos, ya sean personas, instituciones u organizaciones con la finalidad de lograr determinados objetivos profesionales y personales. (15)

Según Cassany, una red social también se define:

[...] como una web formada por los perfiles de todos sus miembros, en los que cada usuario comparte sus datos personales (biografía, intereses, foros, videos) o profesionales (currículum, proyectos, obras) con visibilidad variable (solo para mis amigos, para todos los miembros). (231-233)

Cada miembro selecciona a sus amigos y configura círculos de contactos para compartir el día a día, las lecturas, las canciones, las fotos y los videos que le gustan. Las redes más populares (Facebook, Google, Sonico, Ning) tienen millones de afiliados y son poderosas herramientas para difundir datos y conformar opiniones.

Según Peña et al., las redes sociales son:

[...] sitios web que ofrecen la posibilidad de organizar tu agenda de contactos personales, tu correo electrónico, la mensajería instantánea y, además, te proporcionan espacio para colgar tus fotos, tu músi$\mathrm{ca}$, tus videos, herramientas para gestionar tus grupos de discusión e incluso para crear tu propio blog. (19)

Se conocen como redes sociales a "[...] aquellos sitios web diseñados para asistir a los usuarios en compartir datos personales y revelar sus gustos 
y aficiones, intereses, fotos, con la esperanza de que algún día alguien les permita formar parte de una red social" ("El auge de las redes sociales" 22-24). Sin embargo, las redes sociales son mucho más que simples programas de mensajería o sitios web para conseguir amigos. Estas comunidades funcionan como exclusivos clubes de Internet a los que solamente se puede ingresar por invitación de uno de los miembros ya establecidos, como por ejemplo, en Friendster, Tribe o Bridges.

Las redes sociales son sitios en Internet en las que se crean comunidades de personas con un intercambio dinámico por diferentes motivos:

- Espacios para conocerse, intercambiar ideas, reencontrarse con otras personas.

- Espacios para ofertar productos, servicios y realizar negocios.

- Espacios para compartir e intercambiar información en diferentes medios.

- Espacios para servicios sociales como la búsqueda de personas desaparecidas o intereses particulares.

El valor de estas herramientas radica en la estructura y el modelo comunicativo de todas las herramientas de la Web, como la mensajería instantánea, los chat, los blogs, las videoconferencias. Las redes sociales responden a la necesidad de estar vinculados a otros.

Linda Castañeda afirma que el objetivo principal de una red social:

[...] es conectar sucesivamente a los usuarios que forman parte de esa red. Y qué mejor oportunidad de comunicación interpersonal en la distancia como la red integrada a toda clase de dispositivos electrónicos: portátiles, celulares, tabletas, agendas electrónicas, webcam. (27)

Las redes sociales ofrecen al usuario posibilidades de comunicación y de publicación, como la subida de fotos, el envío de mensajes, los comentarios, la edición de nuestro perfil, comunicarnos de diferentes maneras y en diferentes tiempos.

Linda Castañeda menciona que los usos que se hacen de las redes sociales por parte de los usuarios "[...] son muy variados como de contacto, de comunicación, publicación de fotos, creación de comunidades profesionales. De manera general, se establecen tres grandes grupos de redes sociales: de profesionales, de contacto y especializadas en diferentes ámbitos" (30).
- En los ámbitos académicos, está la web Academia. Para poner a las personas en contacto laboral, existen los sitios King, LinkedIn, Neurona.

- Están las de redes de contacto o generalistas para facilitar la comunicación y poner en contacto personal entre diferentes personas, como Facebook, Tuenti, Orkut, Hi5, Sonico.

- Hay redes sociales especializadas en diferentes ámbitos, como MySpace en la música o Trendtation en la moda. Existen otros sitios que se centran en objetos (fotos, textos, presentaciones) como YouTube o en educación como Educ@contic, Eduteka o Edutopia.

La variedad de las redes especializadas es muy amplia. Según Calvo y Rojas, "[...] cada usuario puede encontrar la red que más se ajuste a sus intereses. Las redes más populares giran alrededor de los siguientes temas: viajes, música, cine, libros, fotografía, video, concursos, animales, bebidas, etnias y rasgos físicos" (73).

Entre las redes sociales más populares por parte de los internautas, según Calvo y Rojas, están por ejemplo (20-21):

1. Facebook: la red social de Mark Zuckerberg se ubicó como la primera dentro de las preferencias de los internautas. Es de fácil uso y permite compartir fotos, videos, ideas y mensajes.

2. Twitter: la red social más utilizada por muchos sectores de la sociedad. Es un servicio gratuito que combina características de los SMs, los blogs y la mensajería instantánea.

3. LinkedIn: es una red social orientada al trabajo. Es una de las mejores cartas de presentación profesional en Internet y es una de las plataformas actuales para hacer negocios y sellar estrategias.

4. Myspace: ofrece grupos, blogs, videos, fotos, mensajería instantánea, etc. Su punto fuerte es la música y el cine.

5. Google Plus: es la integración social de todos los productos de Google. Se trata de una combinación de servicios de diferentes tipos, incluyendo recomendaciones, videochat y mensajería grupal, que abarcan múltiples facetas de nuestras relaciones e interacciones en la web.

Otras redes sociales utilizadas por los internautas son:

1. YouTube: es un sitio web en el cual los usuarios pueden subir y compartir videos. YouTube es un 
sitio de almacenaje gratuito en la red en el que es posible subir para compartir, ver, comentar, buscar y descargar videos. Es uno más de los varios servicios que ofrece Google. Es muy popular gracias a la posibilidad de alojar videos personales de forma sencilla.

2. Instagram: es una aplicación muy popular para dispositivos móviles. Permite editar, retocar y agregarle efectos a las fotos tomadas con los celulares. Ayuda compartir estas imágenes en las redes sociales, y, desde el momento que es posible navegar y explorar las fotos de otros usuarios registrados, se considera una red social.

3. Hi5: está enfocado a un público latinoamericano y ha tenido gran aceptación en México, Perú, Ecuador y Colombia. Fue lanzada en el 2003 y cuenta con más de 70 millones de usuarios.

4. Tagged: es un sitio web masivo que permite a millones de usuarios en el mundo conectarse y generar relaciones. En este puedes compartir tanto mensajes como fotos y videos además de otras interesantes aplicaciones que permiten interactuar con otros usuarios.

5. Tuenti: es una red española a la que se accede por invitación. Tuenti es una red social dedicada a explorar las fotos de usuarios registrados. Una de las características de Tuenti es proteger a toda costa la privacidad de sus usuarios, por lo que es una plataforma cerrada.

\section{Redes sociales en educación}

Para Castañeda, cuando se habla de las redes sociales en relación con la educación, es necesario hacerlo desde tres perspectivas educativas (35):

- Aprender con redes sociales: está vinculado con un aprendizaje formal y uno no formal. Hoy en día los adolescentes están en las redes sociales, por tanto se requiere aprovechar los espacios de interacción y comunicación que se generan entre los jóvenes al formar parte de estas redes desde la educación.

- Aprender a través de redes sociales: se refiere a procesos de aprendizaje informarles en los que los usuarios pueden aprender de manera autónoma e independiente al formar parte de la red.

- Aprender a vivir en un mundo de redes sociales: hace mención a la necesidad de informar y concientizar a los nuevos usuarios sobre estas herramientas, especialmente, acerca de qué son y cómo se usan.

\section{Metodología}

Teniendo en cuenta los objetivos planteados, este estudio fue una investigación-acción. De esta forma, Herreras afirma que la investigación-acción:

[...] supone entender la enseñanza como un proceso de investigación, un proceso de continua búsqueda. Conlleva entender el oficio docente, integrando la reflexión y el trabajo intelectual en el análisis de las experiencias que se realizan, como un elemento esencial de lo que constituye la propia actividad educativa. (1)

De esta forma, esta definición se ajusta a los objetivos planteados en esta investigación, ya que esta se fundamentó en realizar con los extranjeros aprendientes de ELE diversas actividades y ejercicios de lectoescritura de mensajes de texto a través de las redes sociales. Es claro que las observaciones y las correcciones de los ejercicios se orientaron permanentemente por la investigadora.

El método fue inductivo ya que para el desarrollo de los talleres se contó con la participación de cuatro extranjeros que visitaron la Universidad Pedagógica y Tecnológica de Colombia en Tunja. Bernal afirma que el método inductivo "[...] utiliza el razonamiento para obtener conclusiones que parten de hechos particulares aceptados como válidos, para llegar a conclusiones, cuya aplicación sea de carácter general" (56). Así, de acuerdo con el análisis de las actividades realizadas por cada extranjero, se describieron los resultados y las conclusiones. La muestra estuvo constituida por tres estudiantes extranjeros alemanes y una estudiante de la China de 18 a 23 años. Las técnicas fueron los talleres y la observación directa.

Finalmente, el mejoramiento del nivel de lengua en los extranjeros permitió que las actividades en el uso fueran protagónicas tanto en las interacciones directas como en las virtuales. Esto propició ejercicios que involucraran los espacios cibernéticos, como los mensajes de texto en las redes sociales, chat y los blogs.

En relación con la metodología de la investigación se aplicaron talleres a los cuatro aprendientes de ELE. Enseguida se analizaron los resultados provenientes de las aplicaciones de los talleres a los estudiantes de ELE, con el objetivo de replantear los ejercicios y las actividades propuestas. 


\section{Resultados}

Los estudiantes extranjeros realizaron los ejercicios teniendo en cuenta los contenidos de los mensajes de texto de las redes sociales aplicando las instrucciones dadas en Comunicarse en español: la mejor opción (Waked et al.). Así ejercitaban la escritura y la lectura con una interacción social, apropiándose de nuestra cultura a través de Internet, de la telefonía, de los chats, las tabletas y los blogs.

En Comunicarse en español: la mejor opción se presenta, en cada una de las ocho unidades, la sección "comparta sus ideas en la red social". Así, por ejemplo, se estableció que los extranjeros debían realizar los ejercicios considerando las redes sociales y las instrucciones de las ocho unidades. Posteriormente, los aprendientes presentaron los ejercicios realizados por ellos.

Los ejercicios dados a los estudiantes fueron los siguientes:

- En la primera unidad:

Envíe un correo a un amigo e invítelo para que venga y disfrute del aguinaldo boyacense (Waked et al. 18).

- En la segunda unidad:

Envíe un correo electrónico en español a un familiar o amigo que esté en su país natal para que conozca los diferentes ritmos musicales de Colombia (Waked et al. 31).

- En la tercera unidad:

Chatee en una sala de hispanohablantes y establezca una conversación sobre la literatura colombiana (Waked et al. 46).

- En la cuarta unidad:

Entre a un chat e inicie una conversación en español sobre los avances científicos. Escriba las opiniones que más le hayan gustado (Waked et al.65).

- En la quinta unidad:

Por correo electrónico, envíele a un amigo unas fotos de las ciudades colombianas que haya visitado y coméntele qué le ha gustado y qué no de estas (Waked et al. 86).

- En la sexta unidad:

Busque una sala de chat para hispanohablantes y establezca una conversación sobre la vida de los artistas famosos. A continuación presente algunas de las opiniones de sus interlocutores (Waked et al. 101).

- Séptima unidad:

Busque un blog y participe en una discusión sobre el fútbol latinoamericano. Escriba las opiniones de otros participantes opuestas a las suyas (Waked et al. 114).

- Octava unidad:

Reúnase con un grupo de amigos e inicie una conversación sobre los juegos tradicionales de su país. Luego, a través de Skype, muéstrele y explíquele oralmente a un amigo cómo se juega la coca (Waked et al. 125).

Los ejercicios de cada unidad, mencionados anteriormente, fueron realizados por los estudiantes. Estos se evaluaron y analizaron de acuerdo con la escritura, la redacción, la conjugación de los verbos, la cohesión, la coherencia, la interpretación, la gramática y la lectura. Posteriormente, se hicieron las correcciones de cada actividad. En algunas actividades, se encontró que al usar las redes sociales, los extranjeros escribieron algunas palabras abreviadas por la escritura fugaz y rápida.

\section{La comunicación mediante el espacio virtual}

Internet constituye "[...] una forma maravillosa de mostrar al estudiante extranjero recursos y lugares que están fuera del salón de clases como consultar prensa extranjera, gracias a la práctica del idioma como lengua extranjera y al tratamiento de la diversidad multicultural" (Peña et al. 127).

La tecnología móvil e Internet producen nuevos formatos y soportes comunicativos, como el correo electrónico, los mensajes de texto, los chats, los blogs, etc. Estos han propiciado que el espacio virtual se convierta en uno de comunicación que afecta el lenguaje, un ejemplo de lo cual es la escritura. El texto escrito se acomoda a lo virtual y, en algunas ocasiones, afecta el estilo y la ortografía.

La escritura, a pesar de ser un legado cultural, se ha visto inmersa en el uso de las redes sociales. De acuerdo con Carmona y Cortínez, "[...] es un espacio donde el uso frecuente de símbolos y signos ha sustituido en gran parte la 'escritura convencional', con el fin de economizar o suplir a la comunicación no verbal o establecer otro tipo de interacción" (25).

Sin embargo, muchas personas tienen la idea de que "el lenguaje de Internet" incide de forma negativa a los individuos, porque propicia a que las personas deformen las palabras con la intención de agilizar la escritura y la conversación, teniendo en cuenta que aquel uso desmedido de emoticonos se debe al abuso de aplicaciones presentes en las redes sociales (Carmona y Cortínez). 
Cabe resaltar las nuevas prácticas escritoras de los jóvenes en las redes sociales, como las de:

[...] el Facebook, Twitter, [que] rompen los esquemas tradicionales de la escritura estandarizada creando otro tipo de escritura propio de ellos. El hecho que los espacios de escritura del chat sean limitados hace que los jóvenes realicen una serie de modificación al léxico para emitir mensajes rápidos y cortos, dando lugar a la aparición de nuevos signos y códigos. (Vanegas 16)

El correo electrónico se ha convertido en un medio de comunicación vital y de gran éxito en Internet. Actúa como un vehículo eficiente y veloz, y un transmisor de información. El soporte electrónico permite errores ortográficos, de construcción y de léxico. Se puede caracterizar el texto del mensaje electrónico por un estilo informal y por tener alteraciones en la expresión escrita que significan actitudes, emociones, entonaciones. Los extranjeros venían utilizando un contexto virtual en el que predomina la rapidez y la brevedad, pues los aprendientes adaptaron el lenguaje a los ambientes virtuales para alcanzar efectividad y eficacia en la comunicación, lo que dio lugar a la aparición de nuevas formas de expresión escrita.

Posteriormente, se presentaron los ejercicios a los estudiantes extranjeros para que los realizaran mediante el celular, el computador y la tableta teniendo en cuenta las indicaciones dadas.

De esta manera, se detectaron algunos errores en la escritura de los mensajes de la extranjera 4, de la China. Ella escribió algunos mensajes para sus familiares alterando la escritura y cometiendo errores de conjugación y redacción. Por ejemplo: "anteriormente fue [fui] yo que lo hice, si usted está molestada [molesta] con lo que hago. Por qué no me diga [dice], hoy me olvidó hacer a los cuatro estudiantes firmar [me olvide hacer firmar a los cuatro estudiantes] jajaja, jajaja”.

Así mismo, los extranjeros alemanes presentaron algunas alteraciones en la escritura como:

- Repitieron una letra: "holaaaaaa, que tal???????".

- Usaron interjecciones repetidas para transmitir tonos de risa: "jajajaja [risa habitual]".

Utilizaron los emoticones: estos se refieren a un conjunto de símbolos que prefiguran el rostro humano y representan emociones, actitudes o situaciones personales:

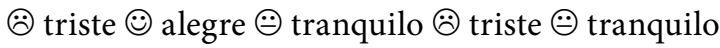

Los aprendientes alemanes utilizaron el servicio de mensajes de textos corto sms, pues es abreviado y simbólico, y han revolucionado el sistema lingüístico con alteraciones en la escritura. Por ejemplo:

- “TQM" (te quiero mucho)

- “KTL??” (¿qué tal?); “onde ands?” (¿por dónde andas?); "1bs fuert" (un beso fuerte).

- “Cmo t va?" (¿cómo te va?); "stas ok?" (¿estás bien?); "ns" (no sé).

- “Xp?” (¿por qué?); "1b” (un beso); “q’hces xq’tn pdido" (¿qué haces?, ¿por qué tan perdido?).

- “A q hr admos?" (¿a qué hora quedamos?)

- “Ola lok ak toy bn q hacs?" (hola, loca, aquí estoy bien: ¿qué haces?

- "Wenas ak stoi bn nsvms dsps salu2" (buenas. Acá estoy bien. Nos vemos después. Saludos).

- “Qndo akba la peli?” (¿cuándo acaba la película?).

- "Hla kmo stas? K ase" (hola, ¿cómo estás?, ¿qué haces?).

- "Yo stoy bn y ud omo está" (yo estoy bien, y ¿usted cómo está?).

- “On tas" (¿dónde estás?).

Se les dieron a los estudiantes otros ejercicios con el fin de corregir los errores evitando la alteración y transformación de la escritura. Cabe aclarar que estas actividades no se encuentran en Comunicarse en español: la mejor opción.

- Envíe un correo electrónico a un familiar o amigo que esté en su país en español a través de Yahoo para que conozca los diferentes ritmos musicales de Colombia.

- Busque un blog y participe escribiendo sus nuevas experiencias en Tunja con sus compañeros extranjeros.

- Encuentre un video en YouTube sobre la presentación de las últimas canciones en español de Shakira y escriba un comentario para sus amigos de habla hispana.

- A través de WhatsApp, mande un escrito o un mensaje de felicitación de cumpleaños a una amiga de habla hispana.

- Envíe a unos amigos unas fotos de la fiesta de la Universidad. Escriba un comentario en español sobre estas en Facebook.

- Utilice el celular para hacer un mensaje de texto a su novia.

- Envíe algunos mensajes de texto a la familia a través de Skype. 
- Mantenga durante un mes un escrito en un blog sobre los equipos de fútbol en Colombia. Escriba a partir de sus intervenciones un correo sobre estos y compártalo.

Finalmente, se describieron los resultados obtenidos de la aplicación de las actividades a los estudiantes extranjeros. En general, los cuatro aprendientes de ELE afirmaron que al desarrollar las actividades pudieron mejorar aspectos gramaticales y aprender vocabulario nuevo. El primer alemán fue muy cuidadoso con la manera de escribir, pues su ortografía y escritura fueron impecables.

En algunas actividades, les faltó profundizar las respuestas dadas. Las respuestas de la segunda aplicación fueron coherentes e interesantes. El segundo alemán le escribió a su familia sobre su estadía en Tunja; le comentó que hay muchas palabras y expresiones nuevas para él que son graciosas. Él prefirió buscar un video de Juanes porque le parece que canta muy bien. En general, su escritura fue buena: utilizó diferentes emoticones para adornar sus escritos. Así mismo, el tercer alemán afirmó que en cada lugar se aprende vocabulario nuevo proveniente de los hablantes, y eso le llamó la atención. Los extranjeros hicieron una comparación entre la selección Colombia y la selección de su país de origen. La estudiante china suele utilizar mucho el WhatsApp: ella le escribe constantemente a su familia y amigos. En la primera aplicación cometió errores de cohesión y conjugación de verbos, pero, con la ayuda de la investigadora, ella y los demás extranjeros mejoraron y fortalecieron la lectura y la escritura en la segunda aplicación de las actividades.

\section{Conclusiones}

De acuerdo con las indicaciones dadas en los ejercicios de Comunicarse en español: la mejor opción, en la sección "Comparta sus ideas en la red social", algunos de los estudiantes extranjeros escribieron mensajes de texto y usaron el lenguaje con dificultad en la ortografía; también tuvieron problemas en la conjugación de verbos; se les hizo difícil que sus ideas estuvieran cohesionadas y fueran coherentes, y se les complicó la redacción y la interpretación. Posteriormente, los cuatro extranjeros desarrollaron otros ejercicios en los que utilizaron las redes sociales mediante la lectoescritura. En estos ejercicios, los extranjeros mejoraron los mensajes de texto, dirigidos a los familiares, amigos, compañeros de estudio y demás personas en una comunicación permanente.

La tecnología móvil e Internet han originado un espacio virtual de comunicación que, en algunas ocasiones, afecta el lenguaje. El soporte electrónico permite que existan errores ortográficos de construcción y de léxico. El desarrollo de los medios tecnológicos y la variedad de textos electrónicos han proporcionado una nueva forma de redacción, caracterizada por la transformación y alteración de la escritura.

Finalmente, este estudio fue un gran aporte para el manejo de la lengua española de los estudiantes, ya que mejoraron los procesos de lectoescritura mediante ejercicios con mensajes de texto en distintas redes sociales. Estos mensajes estuvieron dirigidos a familiares, amigos, compañeros, y se valieron de las intenciones comunicativas que rodean su uso en contextos determinados. Este estudio sirvió también para la estructuración de las unidades y secciones de Comunicarse en español: la mejor opción, especialmente con el pilotaje hecho a los aprendientes alemanes y a la extranjera china, ya que originó la sección "comparta sus ideas con la red social”. Así mismo, ellos mejoraron la competencia comunicativa del español como lengua extranjera durante el desarrollo de los ejercicios y las actividades propuestas.

\section{Referencias}

Bernal-Torres, César. Metodología de la investigación. 2. ${ }^{\mathrm{a}}$ ed. México: Pearson Educación. 2006. Impreso.

Calvo, Montse y Carolina Rojas. Networking: Uso práctico de las redes sociales. Madrid: Esi, 2009. Impreso.

Carmona, Jackeline y Alejandra Cortínez. "Impacto de las redes sociales en el proceso escritural de los jóvenes de la ciudad de Medellín”. Tesis. Universidad de San Buenaventura, 2010. Web. 27 de octubre del 2014. <http:// bibliotecadigital.usbcali.edu.co/jspui/>.

Cassany, Daniel. “En línea”. Leer y escribir en la red. Barcelona: Anagrama, 2012. Impreso.

Castañeda-Quintero, Linda. “Aprendizajes con redes sociales". Tejidos educativos para los nuevos entornos. Bogotá: Eduforma, 2010. Impreso.

“El auge de las redes sociales”. Revista Enter (2004): Ciberespacio. Impreso.

Escandell, María Victoria. Introducción a la pragmática. Barcelona: Ariel, 2002. Impreso. 
Gutiérrez-Ordóñez, Salvador. De pragmática y semántica. España: Arco-libros, 2002. Impreso.

Herreras, Esperanza. "La docencia a través de la investigación-acción". Revista Iberoamericana de Educación (2004). Web. 25 de abril del 2014. <http://www.rieoei. org/deloslectores/682Bausela.PDF>.

Lomas, Carlos, et al. Ciencias del lenguaje, competencia comunicativa y enseñanza de la lengua. Barcelona: Paidós, 1993. Impreso.
Marco Común Europeo de Referencia para las Lenguas: aprendizaje, enseñanza, evaluación. Madrid, España: Ministerio de Educación y Cultura, 2002. Impreso.

Peña, Rosario, et al. Cómo enseñar utilizando las redes sociales. México: Alfaomega, 2013. Impreso.

Peña, Rosario. Nuevas tecnologías en el aula. Bogotá: Ediciones de la U, 2014. Impreso.

Waked, Myriam, Lorena Bernardino-Rodríguez y Gerardo Cardozo. Comunicarse en español: la mejor opción. Tunja: Universidad Pedagógica y Tecnológica de Colombia, 2015. Impreso. 